\title{
COMPARISON OF IN VITRO ACTIVITY OF COLISTIN WITH CEFTOLOZANE/TAZOBACTAM AGAINST MULTI DRUG RESISTANT PSEUDOMONAS AERUGINOSA "A LAST LINE TREATMENT AGAINST MDR"
}

\author{
K. M. Anwarul Islam \\ Associate Professor \\ The Millennium University, Bangladesh \\ Muhammad Aitmaud Uddolah Khan \\ Assistant Professor \\ Baqai Medical University, Pakistan \\ Sarah Azhar \\ Senior Lecturer \\ Baqai Medical University, Pakistan \\ Muhammad Rashid Ahmed \\ Professor \\ Baqai Medical University, Pakistan \\ Sumreen Khurram \\ Lecturer \\ Baqai Medical University, Pakistan \\ Hina Masood \\ Lecturer \\ Baqai Medical University, Pakistan \\ Lubna Farooq \\ Assistant Professor \\ Baqai Medical University, Pakistan
}

\section{Objectives}

\section{ABSTRACT}

To evaluate and compare in vitro activity of Ceftolazane / Tazobactum and Colistin against Multi Drug Resistant (MDR) strains of Pseudomonas aeruginosa.

\section{Methodology}

After ethical approval this in vitro cross-sectional study was conducted from October 2017 to April 2018. Routine samples of pus, wound swabs, blood, tracheal aspirates and urine were collected and received from the in-patient and out-patient clinics. All the samples were submitted for culture and sensitivity testing at the microbiology laboratory of Ziauddin University Hospital, 
North Nazimabad campus. All the samples were processed according to the provided microbiological procedures, CLSI Guidelines 2018.

\section{Results}

Forty sample from the out-patient clinics represented pre-dominance of Multi Drug Resistant strains of Pseudomonas aeruginosa (which was found to be 41.2\%). On culture and sensitivity testing, it was observed that $60 \%$ of the MDR strains of P. aeruginosa were susceptible to Ceftolazane / Tazobactum which was markedly comparable to the susceptibility shown by Colistin (99\%).Statistically, P value was highly significant and was found to be 0.0001 .

\section{Conclusion}

Colistin showed superior activity as compared to Ceftolazane / Tazobactum against MDR isolates of P. aeruginosa. Thus, Colistin has proven to be a possible and important alternative against MDR isolates of $\mathrm{P}$. aeruginosa, but due to its narrow therapeutic window and toxicity profile this drug can be used only when there is no working alternative, or the infection is severely debilitating.

Keywords: Gram-Negative Infection, P. Aeruginosa, Resistant.

\section{INTRODUCTION}

Antibacterial drug are the main therapeutic agents in medicine to treat broad spectrum diseases caused by bacterial infections. In modern science, antibiotic development is one of the milestones. Millions of lives all over the world are saved daily because of the use of antibiotic agents. But there is an important threat round the globe - the emergence of resistance against antibacterial agents (Ventola, 2015).

Irrational and over the counter use of antibiotics result in emergence of resistant isolates of bacteria which do not respond to drug therapy (Buke et al., 2005).

Antibacterial drug resistance threatens this milestone and has become a significant risk to health of mankind. Antibacterial resistance is now a major clinical concern round the globe and is a major public health problem of the current century (Levy, 2002).

$\mathrm{P}$. aeruginosa is a very ubiquitous microorganism, present in many natural and artificial environments (Haley, Colmer-Hamood, \& Hamood, 2012).

P. aeruginosa has a unique grape like odour of aminoacetophenone on culture media / agar (Cox, \& Parker, 1979). It colonizes naturally on skin, in nasal mucosa, throat and also in fecal samples. P. aeruginosa is a nosocomial pathogen. It is also an opportunistic organism and causes life threatening infections in immune-compromised individuals (Kielhofner, Atmar, Hamill, \& Musher, 1992).

According to the data provided by the Center for the Diseases Control and Prevention (CDC), P. aeruginosa can cause a wide variety of infections and is also one of the most common infectious agents for nosocomial pneumonia (Cross et al., 1983), urinary tract infections, ophthalmitis, otitis, surgical wound infections and bacteremia (Trautmann, Halder, Hoegel, Royer, \& Haller, 2008).) Another study revealed that P. aeruginosa is also a leading cause of morbidity and mortality in patients of cystic fibrosis (Kerem, Corey, Gold, \& Levison, 1990).

Colistin is a Polymyxin B antibiotic, which was used as a therapeutic agent for gram negative organisms. (Evans, Feola, \& Rapp, 1999) Due to severe nephrotoxicity and 
neurotoxicity after the therapeutic use of Colistin, its use was diminished (Wolinsky \& Hines, 1962).

Currently due to severe resistance shown by bacteria, Colistin (Polymiyxin B) has reemerged as a last line treatment modality for Multi Drug Resistant isolates of P. aeruginosa, acinetobacter baumannii and Klebseilla pneumonia.

According to our knowledge, in Pakistan no current data is available for usage of $5^{\text {th }}$ generation Cephalosporin comparison with Colistin. Antibiotic resistance, an emerging problem and a clinical concern to treat uropathogenic P.aeruginosa, it is now a high time to look for more specific and sensitive antibacterial agents to treat resistant infections.

The aim of this study is to ascertain the prevalence of P. aeruginosa, in the population of Karachi. Another aim is to determine and compare the sensitivity of Ceftolozane / Tazobactum, a novel $5^{\text {th }}$ generation Cephalosporin. With the sensitivity of Colistin - as it is the last line defense option against Multi Drug Resistant (MDR) strains of P. aeruginosa.

\section{MATERIALS AND METHODS}

This was a Quasi experimental investigation and samples were gathered utilizing nonprobability strategy. Study was directed from October 2017 to April 2018 at Ziauddin University Hospital, Karachi.

Samples were gathered from the inpatient wards and outpatient clinics, subsequently submitted to Microbiology Lab of Ziauddin University Hospital, North Nazimabad campus, Karachi, for culture and sensitivity. Routine samples were taken including pus discharge, wound swabs, blood, tracheal aspirates and urine.

Samples were treated as per microbiological procedures CLSI Guidelines 2018. After an informed and written consent detailed data of the patients and organisms was recorded on a different survey form.

All non-lactose fermenting growths on MacConkey's Agar were collected and stained with Gram stain and biochemical tests (motility, pigment presentation, citrate, catalase and oxidase tests) were run.

Data was scrutinized by utilizing Statistical Package for Social Sciences (SPSS) vesion 21. Descriptive investigations for numerical variables have been referenced as Mean with Standard Deviation.

Frequencies and percentages were determined for susceptibility of antibacterial drugs and MDR strains of $\mathrm{P}$. aeruginosa from different clinical samples. Zones of Inhibition were deciphered according to CLSI Guidelines 2018. Chi Square test was applied to gauge the relationship among sensitivity and resistance patterns of antimicrobial medications. A P-value $<$ 0.05 was considered as factually critical.

\section{RESULTS}

A sum of 176 strains of Pseudomonas was segregated out of which 97 were MDR P. aeruginosa. The pre-dominant bulk of MDR P. aeruginosa segregates were acquired from outpatient division, 40 isolates $(41.2 \%)$ and least were gathered from gynecology ward, 3 isolates $(4.1 \%)$ as appeared in table 2.

It was discovered that $60.0 \%$ of the MDR P. aeruginosa strains were found susceptible to Ceftolozane / Tazobactum but when contrasted with Colistin the sensitivity came out to be $99 \%$. $\mathrm{P}$ value was discovered to be statistically significant $(\mathrm{p}$ value $=0.0001)$ as appeared in table 3. 
Table 1. Gender wise frequency of MDR P. aeruginosa

\begin{tabular}{|l|l|l|c|}
\hline Gender & $\begin{array}{l}\text { MDR } \\
\text { 97(55 \%) }\end{array}$ & $\begin{array}{l}\text { Non MDR } \\
\mathbf{7 9}(\mathbf{4 5 \%})\end{array}$ & P value \\
\hline Male & $45(46 \%)$ & $40(50.6 \%)$ & \multirow{0}{*}{$\mathbf{0 . 5 9}$} \\
\hline Female & $52(54 \%)$ & $37(46.8 \%)$ & \\
\hline
\end{tabular}

Table 2. Percentage of MDR isolates in different departments

\begin{tabular}{|l|l|l|l|}
\hline Department & MDR & Non MDR & P value \\
\hline Gynecology ward & $3(4.1)$ & $0(0.0)$ & \multirow{2}{*}{$\mathbf{0 . 2 9}$} \\
\cline { 1 - 3 } Intensive care unit & $16(16.5)$ & $17(21.5)$ & \\
\cline { 1 - 3 } Surgical ward & $26(27.1)$ & $30(38.0)$ & \\
\cline { 1 - 3 } Outpatient department & $40(41.2)$ & $24(30.4)$ & \\
\cline { 1 - 3 } Medicine ward & $11(11.3)$ & $8(10.1)$ & \\
\hline
\end{tabular}

Table 3. Comparison of susceptibility results of Colistin and $\mathrm{C} / \mathrm{T}$ by disc diffusion method

\begin{tabular}{|l|l|l|l|}
\hline Antibiotics & $\begin{array}{l}\text { Susceptible } \\
\text { N (\%) }\end{array}$ & $\begin{array}{l}\text { Resistant } \\
\text { N }(\%)\end{array}$ & P value \\
\hline $\begin{array}{l}\text { Colistin } \\
\text { Ceftolozane/Tazobactam }\end{array}$ & $\begin{array}{l}99(99 \%) \\
58(60.0 \%)\end{array}$ & $\begin{array}{l}1(1 \%) \\
39(40 \%)\end{array}$ & 0.0001 \\
\hline
\end{tabular}

\section{DISCUSSION}

In immune-compromised patients, diseases such as Pneumonia, Bacteremia, Urinary Tract Infections, Infections of Skin and other soft tissues are usually linked with a notorious / harmful gram negative, aerobic rod-shaped bacillus called Pseudomonas aeruginosa (El Baze et al., 1991).

Clinical isolates of $\mathrm{P}$.aeruginosa show resistance to a lot of classes of antibiotic drugs and have left clinicians with a few choices of course of therapy for infectious diseases (Kalantar, 2012; Rossolini, \& Mantengoli, 2005)

Research studies conducted in different areas/countries of the world also support/exhibit increased prevalence rate of MDR P.aeruginosa. A research performed in India in 2017, at a tertiary care setup demonstrated $85 \%$ prevalence rate of P. aeruginosa (Yuliandra, 2017).

Another study conducted in Egypt showed highest incidence of P.aeruginosa at the burns unit as well as in ICU (Mahmoud, Zahran, Hindawi, Labib, \& Galal, 2013). Another high frequency was also noted in Egypt (56\%) (Farooq, Memon, Ismail, \& Sadiq, 2019).

In Texas a research was conducted in 2010, that revealed a bulk of involvement of MDR P. aeruginosa from gynecology / obstetrics unit (4.1\%), medicine ward (11.3\%), ICU (16.5\%) and from surgical ward $(26.8 \%)$. Another study was conducted and found to support the role of P. aeruginosa in 10 ICU's in France that helped in identifying individual and environmental ICU risk factors that aid in acquiring P. aeruginosa infections (Venier et al., 2014) 
In this study Multi-Drug Resistant strains of P. aeruginosa were found predominantly in the female population and the result was $64 \%$, in comparison to the male population in which the result was $46 \%$. The results are similar to a research conducted in Nepal, which revealed MDR P. aeruginosa strains were (64\%) higher in females while (31\%) lower in males (Kattel, 2012).

Another study on UTI also supported higher incidence of infection by P. aeruginosa in female population as compared to male population. Women aged between 20-40 years show incidence ranging from $25-30 \%$ in contrast to older women aged 60 years or more who showed an incidence ranging from 4 - 43\% (Mittal, Aggarwal, Sharma, Chhibber, \& Harjai, 2009).

In 2013, a cross sectional study was done in a teaching hospital of Ethiopia from January to May and urine samples were collected from 73 patients who were catheterized and developed urinary tract infections. Out of those 73 patients, $P$. aeruginosa was found in urine samples of 36 patients $(49.32 \%)$ who were catheterized, out of which 17 were males $(23.29 \%)$ and 19 were females $(26.03 \%)$. After antibiotic sensitivity testing, all clinical isolates of P. aeruginosa were susceptible to Norfloxacin and Ciprofloxacin. Susceptibility to Gentamicin was also noted and was found to be $86.12 \%$ (Bekele, Tesfaye, Sewunet, \& Waktola, 2015).

Geographical variation and duration of study may also act as an influencing factor that is associated with the difference in gender prevalence.

In our study, it was found that MDR strains of P. aeruginosa are 99\%susceptible to Colistin (Polymyxin B). Another research conducted in Pakistan also supported our study and demonstrated that Colistin (Polymyxin B) is the most sensitive antibacterial option among the antibiotic regimen options (Gill, 2011).

Polymyxin B antibacterial drug, also called Colistin is used as a therapeutic agent against gram negative bacilli. All over the world and in our country Polymyxin B, is a sensitive antibacterial therapeutic agent but due to its narrow therapeutic index and noteworthy side effects, the empirical use of this medicine is very finite (Landersdorfer, \& Nation, 2015).

Colistin was found to have a better in vitro function (98\%) which is significantly comparable to Tazobactum / Ceftalozane (60\%) when diffuse disc method of antibiotic sensitivity was applied.

Increased resistance of bacterial to antibiotic drugs, side effects and toxicities, pharmacokinetics, therapeutic uses, clinical findings and combination therapy in relation to Colistin (Polymyxin B) has been reviewed (Pfaller, Shortridge, Sader, Flamm, \& Castanheira, 2017).

Current methods to utilize Colistin with other antibacterial drugs can be fruitful for increased antibacterial / antibiotic efficacy. In $21^{\text {st }}$ century, it can be predicted that against MultiDrug Resistant gram-negative bacillus - Colistin will be the last line of defense among all antibacterial therapeutic agents (Biswas, Brunel, Dubus, Reynaud-Gaubert, \& Rolain, 2012). As Ceftolazane / Tazobactum is not a drug a choice in our set up, still $40 \%$ resistance was demonstrated in the results. This can be due to cross resistance that is if the patient is resistant to one group of Cephalosporin then cross resistance may develop to newer agents of Cephalosporins.

\section{CONCLUSION}

Resistance of Pseudomonas aeruginosa against antibiotic agents is amplified over a past few years. Previously Colistin was considered a last resort therapy for various Multi Drug Resistant bacteria in clinical set up. Fortunately, Ceftolozane / Tazobactum a comparatively newer antibiotic, the therapeutic use of Ceftolozane / Tazobactum should be reserved only for severe 
and life-threatening infections. This is particularly true where the infection is polymicrobial, anaerobic or Pseudomonas resistant to other antimicrobial drugs.

\section{REFERENCES}

Biswas, S., Brunel, J. M., Dubus, J. C., Reynaud-Gaubert, M., \& Rolain, J. M. (2012). Colistin: an update on the antibiotic of the 21st century. Expert review of anti-infective therapy, 10(8), 917-934.

Bekele, T., Tesfaye, A., Sewunet, T., \& Waktola, H. D. (2015). Pseudomonas aeruginosa isolates and their antimicrobial susceptibility pattern among catheterized patients at Jimma University Teaching Hospital, Jimma, Ethiopia. BMC research notes, 8(1), 488.

Buke, C., Hosgor-Limoncu, M., Ermertcan, S., Ciceklioglu, M., Tuncel, M., Köse, T., Eren, S. (2005). Irrational use of antibiotics among university students. Journal of infection. 51(2), 135-9.

Cox, C. D., \& Parker, J. (1979). Use of 2-aminoacetophenone production in identification of Pseudomonas aeruginosa. Journal of clinical microbiology, 9(4), 479-484.

Cross, A., Allen, J. R., Burke, J., Ducel, G., Harris, A., John, J., ... \& Skalova, R. (1983). Nosocomial infections due to Pseudomonas aeruginosa: review of recent trends. Reviews of infectious diseases, 5(Supplement_5), S837-S845.

Evans, M. E., Feola, D. J., \& Rapp, R. P. (1999). Polymyxin B sulfate and colistin: old antibiotics for emerging multiresistant gram-negative bacteria. Annals of Pharmacotherapy, 33(9), 960-967.

El Baze, P., Thyss, A., Vinti, H., Deville, A., Dellamonica, P., \& Ortonne, J. P. (1991). A study of nineteen immunocompromised patients with extensive skin lesions caused by Pseudomonas aeruginosa with and without bacteremia. Acta dermato-venereologica, $71(5), 411$.

Farooq, L., Memon, Z., Ismail, M. O., \& Sadiq, S. (2019). Frequency and antibiogram of multidrug resistant pseudomonas aeruginosa in a Tertiary Care Hospital of Pakistan. Pakistan journal of medical sciences, 35(6), 1622.

Gill, M. M., Usman, J., Kaleem, F., Hassan, A., Khalid, A., Anjum, R., \& Fahim, Q. (2011). Frequency and antibiogram of multi-drug resistant Pseudomonas aeruginosa. $J$ Coll Physicians Surg Pak, 21(9), 531-534.

Haley, C. L., Colmer-Hamood, J. A., \& Hamood, A. N. (2012). Characterization of biofilm-like structures formed by Pseudomonas aeruginosa in a synthetic mucus medium. BMC microbiology, 12(1), 181.

Kielhofner, M., Atmar, R. L., Hamill, R. J., \& Musher, D. M. (1992). Life-threatening Pseudomonas aeruginosa infections in patients with human immunodeficiency virus infection. Clinical infectious diseases, 14(2), 403-411.

Kerem, E., Corey, M., Gold, R. T., \& Levison, H. (1990). Pulmonary function and clinical course in patients with cystic fibrosis after pulmonary colonization with Pseudomonas aeruginosa. The Journal of pediatrics, 116(5), 714-719.

Kalantar, E., Torabi, V., Salimizand, H., Soheili, F., Beiranvand, S., \& Dallal, M. M. S. (2012). First Survey of metallo- $\beta$-lactamase producers in clinical isolates of Pseudomonas aeruginosa from a referral burn center in Kurdistan province. Jundishapur journal of natural pharmaceutical products, 7(1), 23. 
Kattel, H. P., Mishra, S. K., Acharya, J., Sigdel, M. R., Prasad, N., Shah, A. S. S., ... \& Pokhrel, B. M. (2012). Antibiotic sensitivity profile of different uropathogens in a tertiary care center in Nepal. Journal of Nepal Association of Medical Laboratory Science, 11(1), 1933.

Levy, S. B. (2002). Factors impacting on the problem of antibiotic resistance. Journal of Antimicrobial Chemotherapy, 49(1), 25-30.

Landersdorfer, C. B., \& Nation, R. L. (2015, February). Colistin: how should it be dosed for the critically ill?. In Seminars in respiratory and critical care medicine (Vol. 36, No. 01, pp. 126-135). Thieme Medical Publishers.

Mahmoud, A. B., Zahran, W. A., Hindawi, G. R., Labib, A. Z., \& Galal, R. (2013). Prevalence of multidrug-resistant Pseudomonas aeruginosa in patients with nosocomial infections at a university hospital in Egypt, with special reference to typing methods. J Virol Microbiol, 13, 165-59.

Mittal, R., Aggarwal, S., Sharma, S., Chhibber, S., \& Harjai, K. (2009). Urinary tract infections caused by Pseudomonas aeruginosa: a minireview. Journal of infection and public health, 2(3), 101-111.

Pfaller, M. A., Shortridge, D., Sader, H. S., Flamm, R. K., \& Castanheira, M. (2017). Ceftolozane-tazobactam activity against drug-resistant Enterobacteriaceae and Pseudomonas aeruginosa causing healthcare-associated infections in Australia and New Zealand: Report from an Antimicrobial Surveillance Program (2013-2015). Journal of global antimicrobial resistance, 10, 186-194.

Rossolini, G. M., \& Mantengoli, E. (2005). Treatment and control of severe infections caused by multiresistant Pseudomonas aeruginosa. Clinical Microbiology and infection, 11, 1732.

Trautmann, M., Halder, S., Hoegel, J., Royer, H., \& Haller, M. (2008). Point-of-use water filtration reduces endemic Pseudomonas aeruginosa infections on a surgical intensive care unit. American journal of infection control, 36(6), 421-429.

Venier, A. G., Leroyer, C., Slekovec, C., Talon, D., Bertrand, X., Parer, S., ... \& Clair, B. (2014). Risk factors for Pseudomonas aeruginosa acquisition in intensive care units: a prospective multicentre study. Journal of Hospital Infection, 88(2), 103-108.

Ventola, C. L. (2015). The antibiotic resistance crisis: part 1: causes and threats. Pharmacy and therapeutics, 40(4), 277.

Wolinsky, E., \& Hines, J. D. (1962). Neurotoxic and nephrotoxic effects of colistin in patients with renal disease. New England Journal of Medicine, 266(15), 759-762.

Yuliandra, Y. (2017). Antibacterial Resistance Pattern of Pseudomonas Aeruginosa Isolated from Clinical Samples at A General Hospital in...

\section{Copyrights}

Copyright for this article is retained by the author(s), with first publication rights granted to the journal. This is an open-access article distributed under the terms and conditions of the Creative Commons Attribution license (http://creativecommons.org/licenses/by/4.0/) 